\title{
ON THE ESTIMATION OF PARAMETERS IN THE STATISTICAL PREDICTION OF EARTHQUAKES
}

\author{
So Gu KIM \\ Department of Physics, Hanyang University, Seoul, Korea \\ (Received June 11, 1983; Revised November 22, 1983)
}

\begin{abstract}
The modified first and third asymptotic functions of the extreme value theory are applied to compute return periods and maximum potential earthquakes in densely populated regions such as the Tokyo, Guatemalan, Managuan, and Los Angeles areas.

The plot of the modified first asymptotic distribution follows an upward convex curve on Gumbel probability paper and is almost identical to that of the third asymptotic distribution. An application of the third asymptotic function shows too large maximum potential earthquakes to be realistic in view of the finiteness of the seismic source, whereas the modified first asymptotic function gives more reasonable maximum potential earthquakes and more optimum evaluation in seismic risk than the third one.
\end{abstract}

\section{Introduction}

The statistical theory of extreme value for earthquakes has been used by many seismologists (LOMNITZ, 1966; KÁRNìK and HÜBERNOVÁ, 1968; SHAKAL and WILlis, 1972; Ch'EN and LIN, 1973; Yegulalp and Kuo, 1974; Hattorr, 1979; KIM, 1981). They employed the first and third asymptotic distributions of the largest values originated by GUMBEL (1958) to estimate the probable occurrence of the maximum potential earthquake. SHAKal and WiLlis (1972) followed the first distribution function, whereas YeGulalP and Kuo (1974) and HATTORI (1979) preferred employing the third distribution function for probabilistic models. CH'EN and LIN (1973) have modified the first distribution function by assuming that the earthquake magnitude of each region has an upper limit of the maximum potential earthquake. In this study, a Taylor expansion was adopted to the modified first and the third asymptotic functions to compute the predictive parameters of extreme earthquakes. The computation procedure is iterated until the convergent values of approximate parameters are found to be finite. It is also found that the solution of the third asymptotic function of the author is identical with that of YeGULALP and KUO (1974) and HATTORI (1979).

These two asymptotic functions are applied to compute return periods and maximum potential earthquakes in densely populated areas such as the Tokyo $\left(35^{\circ}-36^{\circ} \mathrm{N}, 139^{\circ}-140^{\circ} \mathrm{E}\right)$, Guatemalan $\left(14^{\circ}-16^{\circ} \mathrm{N}, 89^{\circ}-92^{\circ} \mathrm{W}\right)$, Managuan $\left(11^{\circ}-\right.$ $13^{\circ} \mathrm{N}, 85^{\circ}-87^{\circ} \mathrm{W}$ ), and Los Angeles $\left(33^{\circ}-35^{\circ} \mathrm{N}, 117^{\circ}-119^{\circ} \mathrm{W}\right)$, The results of 
the first modified distribution function can be compared with those of the third asymptotic distribution to provide a means for testing their goodness-of-fit.

\section{Basic Theory of Largest Values}

In seismology the analysis of extremes is often applied to the time series of magnitudes within a region, or intensities observed at a certain point. The extremes of the series are obtained by dividing the time period into equal sections and extracting the maximum value for each interval. For a given time interval, the asymptotic distributions of extremes for the first and the third types (GuMBEL, 1958) are given by

$$
\begin{gathered}
G^{1}(x)=\exp \left[-\mathrm{e}^{-\beta(x-w)}\right], \quad \beta>0 \\
G^{3}(x)=\exp \left[-\left(\frac{w-x}{w-u}\right)^{k}\right], \quad k>0, x \leq w, u<w
\end{gathered}
$$

where $u$ is the characteristic largest value, $\beta$ is the extremal intensity function, $k$ is the shape parameter, and $w$ is the upper limit of the largest values.

For a time series of events, Eq. (1) contains unlimited values of $x$ and Eq. (2) contains upper limits of the maximum potential earthquakes. The values $G(x)$ for various values of $x$ can be found as follows. The time series is divided into equal time intervals and the largest value of $x$ found in each case. This gives a series of $N$ extremes which are then ranked in ascending order of size $x$. For the $j$-th largest value $x_{j}$, an estimate of $G\left(x_{j}\right)$ is

$$
G\left(x_{j}\right)=\frac{j}{N+1}
$$

where $j=1,2, \ldots, N, N$ is the number of observations. If all the values of $x_{j}$ are available, the application of Eq. (3) is simple. In case, however, either no earthquakes occurred during $n$ years, or earthquakes with a magnitude of less than 5 did occur but were missed, analysis is made assuming that the data period is available for $(N-n)$. In such a case, only $x_{n}+\cdots x_{N}$ are plotted on Gumbel probability paper. As a consequence, an estimate of $G\left(x_{j}\right)$ is

$$
G\left(x_{j}\right)=\frac{j+n}{N+1},
$$

where $j=1,2, \ldots, N-n, n$ is missing data and $N$ is the total data period.

If the distribution is of the first type, the plots lie on a straight line. On the other hand, if the distribution is of the third type, the plot follows an upward convex curve.

$\mathrm{CH}^{+} \mathrm{EN}$ and LIN (1973) modified the first type of distribution function to have the upper limit of largest value as an upward convex curve. They have derived a new distribution function of extreme values from the first asymptotic function by assuming that the earthquake magnitude of each earthquake region has an 
upper limit and therefore does not tend to be infinite. The modified first asymptotic distribution of extreme values is defined by

$$
\begin{gathered}
G(x)=\exp \left(-\mathrm{e}^{\beta u} \cdot \frac{\mathrm{e}^{-\beta x}-\mathrm{e}^{-\beta v}}{1-\mathrm{e}^{-\beta v}}\right), \quad \beta>0, \quad v>u>0 \\
G(x)=\exp \left[-\mathrm{e}^{-\beta(x-u)}\left\{1-\mathrm{e}^{-\beta(v-x)}\right\}\right], \quad \mathrm{e}^{-\beta v} \ll 1 .
\end{gathered}
$$

The value $v$ is the upper limit of the magnitude of earthquakes occurring in a certain earthquake region. When earthquake magnitude is relatively small, the term $\mathrm{e}^{-\beta(v-x)}$ from the avobe equation becomes close to zero and the forms of the first and the modified first asymptotic functions remain the same, which indicates that $x$ and $-\ln [-\ln G(x)]$ still show a linear relationship. When earthquake magnitude is relatively large, the curve representing a relationship between $x$ and $-\ln [-\ln G(x)]$ follows an upward convex curve tending towards the extreme value of $v$. This explains that the modified first asymptotic function has the same physical meaning for a probabilistic model as the third asymptotic function. Furthermore, previous observations indicate that the earthquake magnitude of each earthquake region must have an upper limit when time tends toward infinity. The results show that the modified first and third asymptotic functions are more plausible than the first type.

The time intervals are selected arbitrarily to determine the extremes, but should be long enough for all the extremes to be above the lower threshold value for completeness of the data file. By using annual extremes, the return period than an extreme value equal to or exceeding $x$ occurs is given by,

$$
T(x)=\frac{1}{1-G(x)}
$$

\section{Estimation of Parameters}

Since Eqs. (2) and (5) are not linear, it becomes necessary to apply the method of Taylor expansion to formulate the simultaneous linear equations. YeGulalP and Kuo (1974), however, estimated parameters by using a direct approach of the least square which is also computed for comparison. Taking the logarithm of both sides of Eq. (2) twice,

$$
y_{j}=-\ln [-\ln G(x)]=-k \ln \left(\frac{w-x_{i}}{w-u}\right) .
$$

Equations (2) and (5) are expanded by Taylor series about the points $\left(k_{0}, u_{0}, w_{0}\right)$ and $\left(\beta_{0}, u_{0}, v_{0}\right)$ that are obtained by the initial guess. Taking only the first-order terms into account from the Taylor expansion of Eq. (8),

$$
\begin{aligned}
y_{j}= & f\left(x_{j} ; k, u, w\right)=-\left.k \ln \left(\frac{w-x_{j}}{w-u}\right) \simeq f\right|_{0}+\left.\frac{\partial f}{\partial k}\right|_{0}(\Delta k) \\
& +\left.\frac{\partial f}{\partial u}\right|_{0}(\Delta u)+\left.\frac{\partial f}{\partial w}\right|_{0}(\Delta w)+\cdots,
\end{aligned}
$$


where

$$
\begin{gathered}
\left.f\right|_{0}=-k_{0} \ln \left(\frac{w_{0}-x_{j}}{w_{0}-u_{0}}\right),\left.\quad \frac{\partial f}{\partial k}\right|_{0}=-\ln \left(\frac{w_{0}-x_{j}}{w_{0}-u_{0}}\right) \\
\left.\frac{\partial f}{\partial u}\right|_{0}=-\frac{k}{\left(w_{0}-u_{0}\right)},\left.\quad \frac{\partial f}{\partial w}\right|_{0}=\frac{k_{0}\left(u_{0}-x_{j}\right)}{\left(w_{0}-x_{j}\right)\left(w_{0}-u_{0}\right)} \\
y_{j}-\left.f\right|_{0}=-\ln \left(-\ln \frac{j}{N+1}\right)+k_{0} \ln \left(\frac{w_{0}-x_{j}}{w_{0}-u_{0}}\right) .
\end{gathered}
$$

Similarly Eq. (5) becomes

$$
y_{j}=-\ln [-\ln G(x)]=\beta\left(x_{j}-u\right)-\ln \left[1-\mathrm{e}^{-\beta\left(v-x_{j}\right)}\right]+\ln \left(1-\mathrm{e}^{-\beta v}\right) .
$$

Taking only the first-order terms of the Taylor expansion of Eq. (10).

$$
y_{j}=\left.g\left(x_{j} ; \beta, u, v\right) \simeq g\right|_{0}+\left.\frac{\partial g}{\partial \beta}\right|_{0}(\Delta \beta)+\left.\frac{\partial g}{\partial u}\right|_{0}(\Delta u)+\left.\frac{\partial g}{\partial v}\right|_{0}(\Delta v)+\cdots,
$$

where

$$
\begin{gathered}
\left.g\right|_{0}=\beta_{0}\left(x_{j}-u_{0}\right)-\ln \left[1-\mathrm{e}^{-\beta_{0}\left(v_{0}-x_{j}\right)}\right]+\ln \left(1-\mathrm{e}^{-\beta_{0} v_{0}}\right) \\
\left.\frac{\partial g}{\partial \beta}\right|_{0}=\left(x_{j}-u_{0}\right)-\frac{\left(v_{0}-x_{j}\right) \mathrm{e}^{-\beta_{0}\left(v_{0}-x_{j}\right)}}{1-\mathrm{e}^{-\beta_{0}\left(v_{0}-x_{j}\right)}}+\frac{v_{0}}{\mathrm{e}^{\beta_{0} v_{0}}-1} \\
\left.\frac{\partial g}{\partial u}\right|_{0}=-\beta_{0} \\
\left.\frac{\partial g}{\partial v}\right|_{0}=\frac{-\beta_{0} \mathrm{e}^{-\beta_{0}\left(v_{0}-x_{j}\right)}}{1-\mathrm{e}^{-\beta_{0}\left(v_{0}-x_{j}\right)}}+\frac{\beta_{0}}{\mathrm{e}^{\beta_{0} v_{0}}-1} \\
y_{j}-\left.g\right|_{0}=-\ln \left(-\ln \frac{j}{N+1}\right)-\beta_{0}\left(x_{j}-u_{0}\right)+\ln \left[1-\mathrm{e}^{-\beta_{0}\left(v_{0}-x_{j}\right)}\right]-\ln \left(1-\mathrm{e}^{-\beta_{0} v_{0}}\right) .
\end{gathered}
$$

The initial values are obtained from the first asymptotic function of Eq. (1), except for $v$ which can be obtained by adding a magnitude 0.5 or 1 to the largest earthquake that has already occurred. The initial values for the third asymptotic function are estimated by the modified first asymptotic function for $1<k<10$. Substituting these initial values into Eqs. (8) and (10), the least square method and Gauss elimination are used to determine $\Delta k, \Delta u, \Delta w, \Delta \beta$, and $\Delta v$. By adding the correction values $\Delta k, \Delta \beta, \Delta u, \Delta w$, and $\Delta v$ to the initial values $k_{0}, \beta_{0}, u_{0}, w_{0}$, and $v_{0}$ the required values are obtained by

$$
\begin{gathered}
k=k_{0}+\Delta k \\
\beta=\beta_{0}+\Delta \beta \\
u=u_{0}+\Delta u \\
w=w_{0}+\Delta w \\
v=v_{0}+\Delta v .
\end{gathered}
$$

The above values are used again as input factors for iterative purposes and new approximate equations are made successively for consecutive computation of 
a set of correction values. The computation procedure of the correction values is repeated until the solution converges (KIM, 1978).

The statistical parameters for recurrence of earthquakes are estimated by using the above theory of extremes. The exceeding probability of any given magnitude $\geq x$ in $t$ years is given by

$$
p(M \geq x)=1-\exp \left[-\frac{t}{T(x)}\right]
$$

The maximum magnitude with an exceeding probability in $t$ years is

$$
V_{t}=-\frac{1}{\beta} \ln [\exp (-\beta v)+\exp (z-\beta u)], \quad z=\ln [-\ln G(x)]
$$

for the modified first asymptotic function and

$$
W_{t}=w-(w-u) \exp \left(\frac{z}{k}\right)
$$

for the third asymptotic function.

It is useful to point out that using Eq. (13) and setting $t=T(X), P$ is found to equal 0.63 or $63 \%$. Therefore, if the time period is equal to the return period, the acceleration or magnitude will have a 63 percent probability of being exceeded within the given time period.

\section{Application and Results}

The extreme value theory is quite often applied to statistical prediction of earthquakes as well as seismic risk evaluation in earthquake engineering. YeGULALP and Kuo (1974) applied the third asymptotic function to world-wide seismicity by assuming that (1) the prevailing conditions must be valid in the future, and (2) the observed largest values are independent of each other. SHAKAL and WiLLIs (1972) used the first type of asymptotic function to estimate probabilities. CH'EN and LIN (1973) developed the modified form of the first asymptotic function to compute the mean return periods and the maximum potential earthquakes in China. HATTORI (1979) employed the third asymptotic function to make seismic risk maps of certain areas of the world. They all indicated that there is a special advantage in applying the extreme value theory to statistical analysis of earthquake data since it requires only comparatively large shocks within a given time interval. Furthermore, application of the extreme value theory to historical earthquakes is of great importance since small earthquakes are often missed.

In this paper, the modified first and third asymptotic distributions are used to compute return periods and maximum potential earthquakes for densely populated regions such as the Tokyo-Kamakura $\left(35^{\circ}-36^{\circ} \mathrm{N}, 139^{\circ}-140^{\circ} \mathrm{E}\right)$, Guatemalan $\left(14^{\circ}-16^{\circ} \mathrm{N}, 89^{\circ}-92^{\circ} \mathrm{W}\right)$, Managuan $\left(11^{\circ}-13^{\circ} \mathrm{N}, 85^{\circ}-87^{\circ} \mathrm{W}\right)$, and Los 


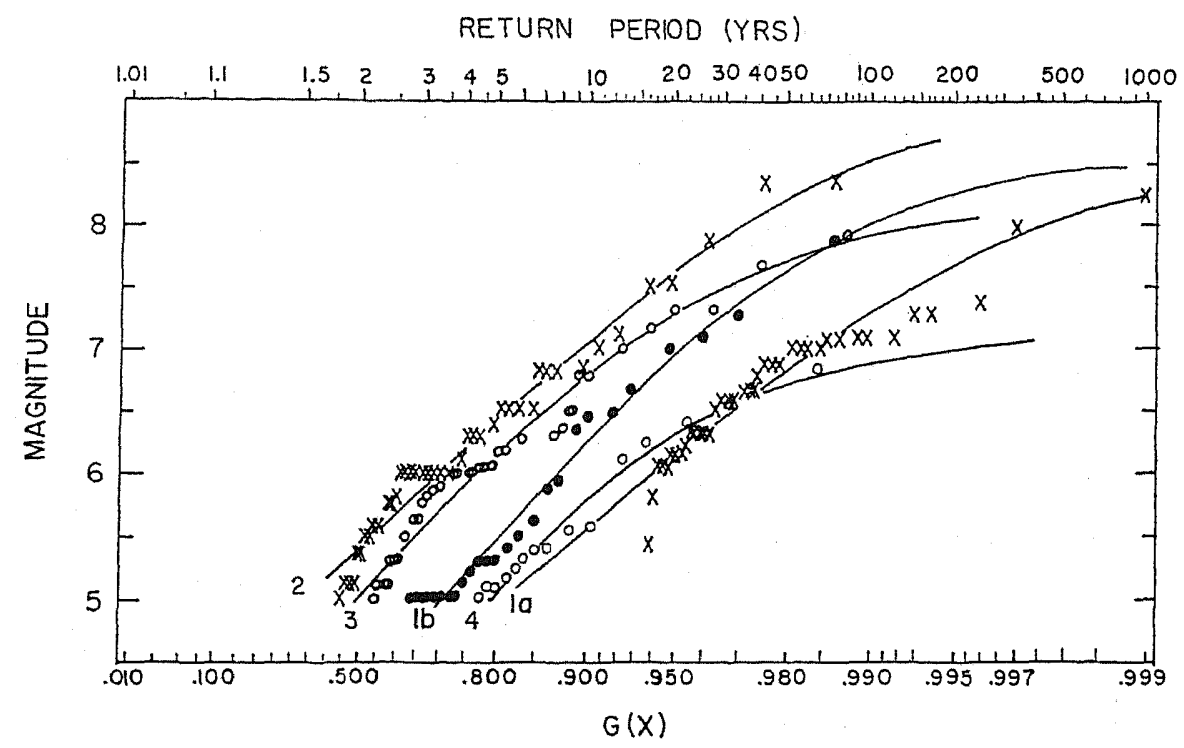

Fig. 1. The modified first asymptotic distribution and observed maximum magnitudes. 1a, the Tokyo region for the period 1213-1931; 1b, the Tokyo region for the period 1906-1979; 2, the Guatemalan region; 3, the Managuan region; 4, the Los Angeles region.

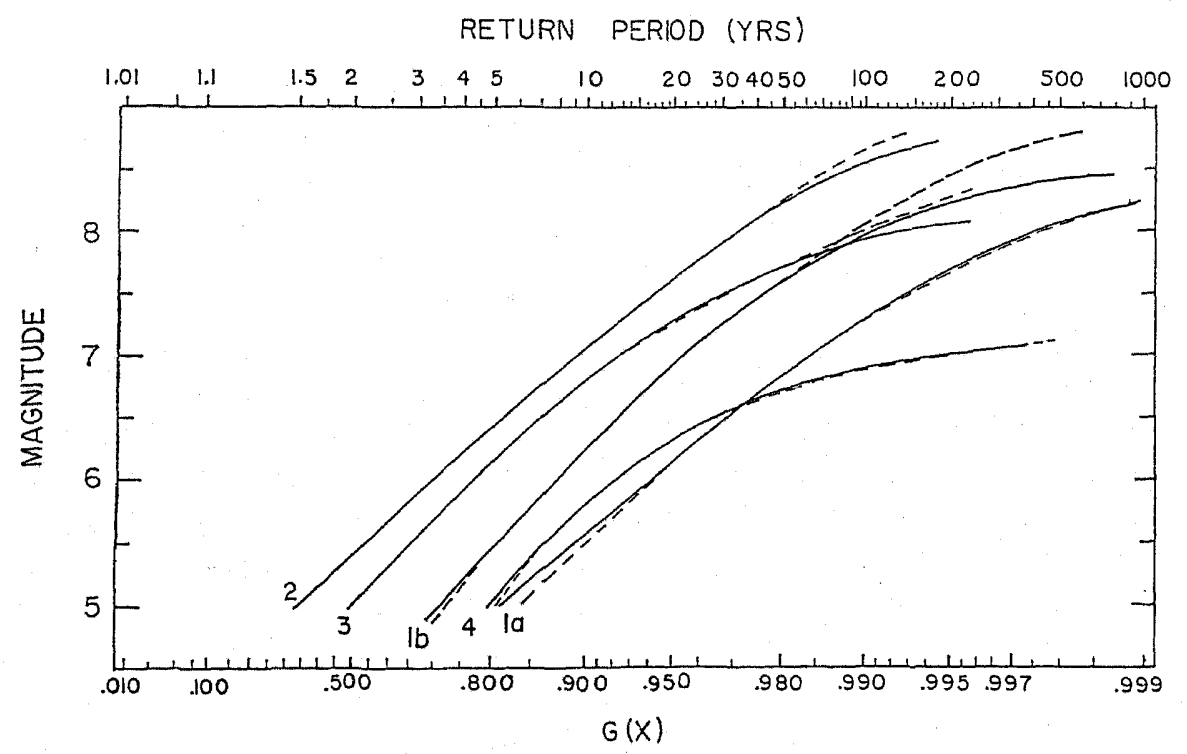

Fig. 2. Comparison of asymptotic distribution curves for the modified first and third asymptotic functions. - indicates the modified first type, --- indicates the third type. 
Table 1. Maximum earthquake magnitudes of selected years in the order of increasing magnitude.

\begin{tabular}{ccccccccc}
\hline \multicolumn{2}{c}{$\begin{array}{c}\text { Tokyo* } \\
1213-1931\end{array}$} & \multicolumn{2}{c}{$\begin{array}{c}\text { Tokyo } \\
\text { 1906-1979 }\end{array}$} & \multicolumn{2}{c}{$\begin{array}{c}\text { Guatemala } \\
1902-1979\end{array}$} & \multicolumn{2}{c}{$\begin{array}{c}\text { Managua } \\
1898-1979\end{array}$} & $\begin{array}{c}\text { Los Angeles } \\
1916-1979\end{array}$ \\
\hline 5.4 & 6.7 & 5.0 & 5.4 & 5.0 & 6.0 & 5.0 & 6.0 & 5.0 \\
5.7 & 6.7 & 5.0 & 5.5 & 5.1 & 6.0 & 5.1 & 6.1 & 5.1 \\
6.1 & 6.8 & 5.0 & 5.6 & 5.1 & 6.1 & 5.1 & 6.1 & 5.1 \\
6.1 & 6.9 & 5.0 & 5.88 & 5.1 & 6.25 & 5.1 & 6.13 & 5.2 \\
6.1 & 6.9 & 5.0 & 5.9 & 5.4 & 6.25 & 5.3 & 6.2 & 5.25 \\
6.2 & 6.9 & 5.0 & 6.4 & 5.4 & 6.25 & 5.3 & 6.2 & 5.3 \\
6.2 & 7.0 & 5.0 & 6.5 & 5.5 & 6.4 & 5.3 & 6.25 & 5.4 \\
6.2 & 7.0 & 5.0 & 6.5 & 5.5 & 6.5 & 5.5 & 6.3 & 5.4 \\
6.3 & 7.0 & 5.1 & 6.7 & 5.6 & 6.5 & 5.6 & 6.38 & 5.5 \\
6.4 & 7.0 & 5.2 & 7.0 & 5.6 & 6.5 & 5.6 & 6.5 & 5.5 \\
6.4 & 7.1 & 5.3 & 7.1 & 5.7 & 6.5 & 5.7 & 6.5 & 6.25 \\
6.4 & 7.1 & 5.3 & 7.2 & 5.75 & 6.75 & 5.75 & 6.75 & 6.3 \\
6.4 & 7.1 & 5.3 & 7.9 & 5.75 & 6.75 & 5.8 & 6.75 & 6.4 \\
6.4 & 7.1 & & & 5.8 & 6.75 & 5.88 & 7.0 & 6.5 \\
6.4 & 7.1 & & & 6.0 & 6.75 & 6.0 & 7.2 & 6.8 \\
6.5 & 7.2 & & & 6.0 & 7.0 & 6.0 & 7.3 & \\
6.6 & 7.2 & & & 6.0 & 7.1 & 6.0 & 7.3 & \\
6.6 & 7.3 & & & 6.0 & 7.5 & 6.0 & 7.7 & \\
6.6 & 7.9 & & & 6.0 & 7.5 & & 7.9 & \\
6.6 & 8.2 & & & 6.0 & 7.9 & & & \\
6.7 & & & & 6.0 & 8.3 & & & \\
& & & & & 8.3 & & & \\
6 & & & & & & &
\end{tabular}

* Historical earthquakes.

Table 2. Estimation of statistical parameters in earthquake prediction.

\begin{tabular}{cllllll}
\hline Region & Type & $\beta$ & $k$ & $u$ & $v$ & $w$ \\
\hline 1a. Tokyo & MF & 1.24 & & 3.78 & 8.5 & \\
(1213-1931) & TH1 & & 4.04 & 2.48 & & 9.5 \\
& TH2 & & 4.04 & 2.48 & & 9.5 \\
\hline 1b. Tokyo & MF & 0.85 & & 3.75 & 8.6 & \\
(1906-1979) & TH1 & & 5.16 & 3.47 & & 11.3 \\
& TH2 & & 5.12 & 3.44 & & 11.3 \\
\hline 2. Guatemala & MF & 1.07 & & 5.00 & 9.1 & \\
& TH1 & & 8.50 & 4.94 & & 13.8 \\
& TH2 & & 8.57 & 4.96 & & 13.9 \\
\hline 3. Managua & MF & 0.94 & & 4.67 & 8.2 & \\
& TH1 & & 3.66 & 4.46 & & 9.4 \\
& TH2 & & 3.66 & 4.46 & & 9.4 \\
\hline 4. Los Angeles & MF & 0.58 & & 3.02 & 7.1 & \\
& TH1 & & 1.54 & 1.22 & & 7.2 \\
& TH2 & & 1.55 & 1.47 & & 7.2 \\
\hline
\end{tabular}

MF is the modified first asymptotic function. TH1 and TH2 are the third asymptotic functions solved by the author (TH1) and YEGULALP and KUO (1974) or HATTORI (1979). 


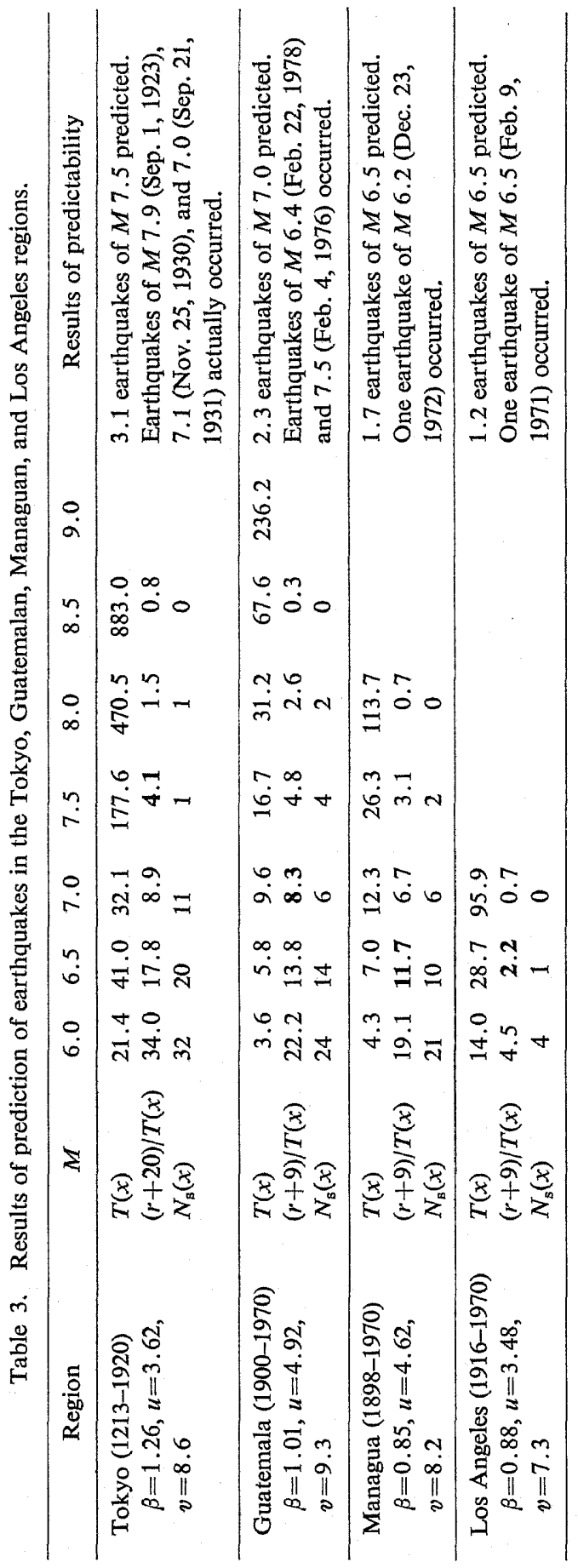


Angeles $\left(33^{\circ}-35^{\circ} \mathrm{N}, 117^{\circ}-119^{\circ} \mathrm{W}\right)$ areas. According to recent figures 142,807 persons (World MAP of Natural Hazards, 1978) lost their lives (in Tokyo and Yokohama) in the Great Kanto earthquake of September 1, 1923. The Guatemalan earthquake of February 4, 1976 caused 22,778 deaths in central Guatemala. The Managuan earthquake of December 23, 1972 killed 5,000 in Managuan and the San Fernando earthquake of February 9, 1971 killed 65 in San Fernando. Therefore it is worth-while to find the statistical parameters of earthquake prediction in these regions.

In estimating earthquake magnitudes, most large or old events are based on Richter magnitudes or surface-wave magnitudes, whereas small or recent ones depend primarily upon body-wave magnitudes. In the case of the Tokyo region (1213-1931), large uncertainties ( \pm 1 magnitude unit) may be introduced by using historical earthquakes. All the data of instrumental earthquakes is assumed to have an uncertainty of \pm 0.5 magnitude unit. Information concerning instrumental earthquakes is provided by NOAA, and that concerning historical earthquakes in the Tokyo region (1213-1931) is extracted from UsAMI (1974). As a result, historical data for the Tokyo region does not fit the upward convex curve of Fig. 1.

The hyperbolic curves of the two asymptotic distributions are significantly consistent with each other for goodness-of-fit for the extreme distributions in Fig. 2. Notwithstanding, there are some physical and computational difference between the modified first and third asymptotic methods. The maximum potential earthquakes determined by the third asymptotic distribution are often too large to be realistically observed in seismology.

The maximum potential earthquakes for the Tokyo region in the periods 12131931 and 1906-1979, Guatemalan, Managuan, and Los Angeles regions are found to be $8.5,8.6,9.1,8.2$, and 7.1 using the modified first type, 9.5, 11.3, 13.8, 9.4, and 7.2 using the third type calculated by the author, and 9.5, 11.3, 13.9, 9.4, and 7.2 using the third type of Yegulalp and Kuo or Hattori. YegulalP and Kuo (1974) have determined unusually large maximum potential earthquakes using the third type function from the data (for example, New Hebrides Islands and Mariana Islands). The fact that the largest earthquakes reported in the worldwide catalog have $M_{\mathrm{s}}=8.9$ or thereabouts (DUDA, 1965) indicates that there must be a finite upper bound to the largest earthquake. In the light of maximum potential earthquakes, the modified first asymptotic function is more reasonable than the third one. As shown in Table 2, it is obvious that an application of the third asymptotic function results in a finite chance that a $M>9$ event will appear in a computer-generated list of earthquakes if the list is of sufficient length. Therefore, the modified first asymptotic distribution is preferable to the third one as a general model concerning the physical size of maximum magnitude earthquakes in seismology.

In Table $3, N_{\mathrm{s}}(x)$ is the number of earthquakes that actually occurred within the period. One may compare $N_{\mathrm{s}}(x)$ with the statistical average of earthquakes 
during the $r$ years, $N_{\mathrm{r}}(x)=r$, to check the possibility of the statistical prediction. during the $r$ years, $N_{\mathrm{r}}(x)=(r) / T(x)$, to check the possibility of the statistical prediction. If $N_{\mathrm{r}}(x)-N_{\mathrm{s}}(x) \geq 1$, it indicates that the number of earthquakes which actually occurred within the given period is less than that of earthquakes which should be occurring within $r$ years. Consequently, the probability of earthquake danger increases. An application of the statistical mean period, $T(x)$, may be extended to the next $r^{\prime}$ years by representing the number of earthquakes that would occur in the next $r^{\prime}$ years as $\left(r^{\prime}\right) / T(x)$. The line, $(r+20) / T(x)$, represents the number of earthquakes that should occur within $r+20$ years, or the prediction of earthquakes for the next 20 years. It is found that return periods of the Tokyo region for the period 1213-1931 are respectively greater than for the period 19061979, since the magnitudes of historical earthquakes are determined on the basis of subjective observation of large earthquakes.

Earthquake prediction assumes that the behavior of the maximum magnitude earthquakes in the future will be similar to that of past maximum earthquakes. Therefore, the number of earthquakes that are likely to occur in the next $n$ years

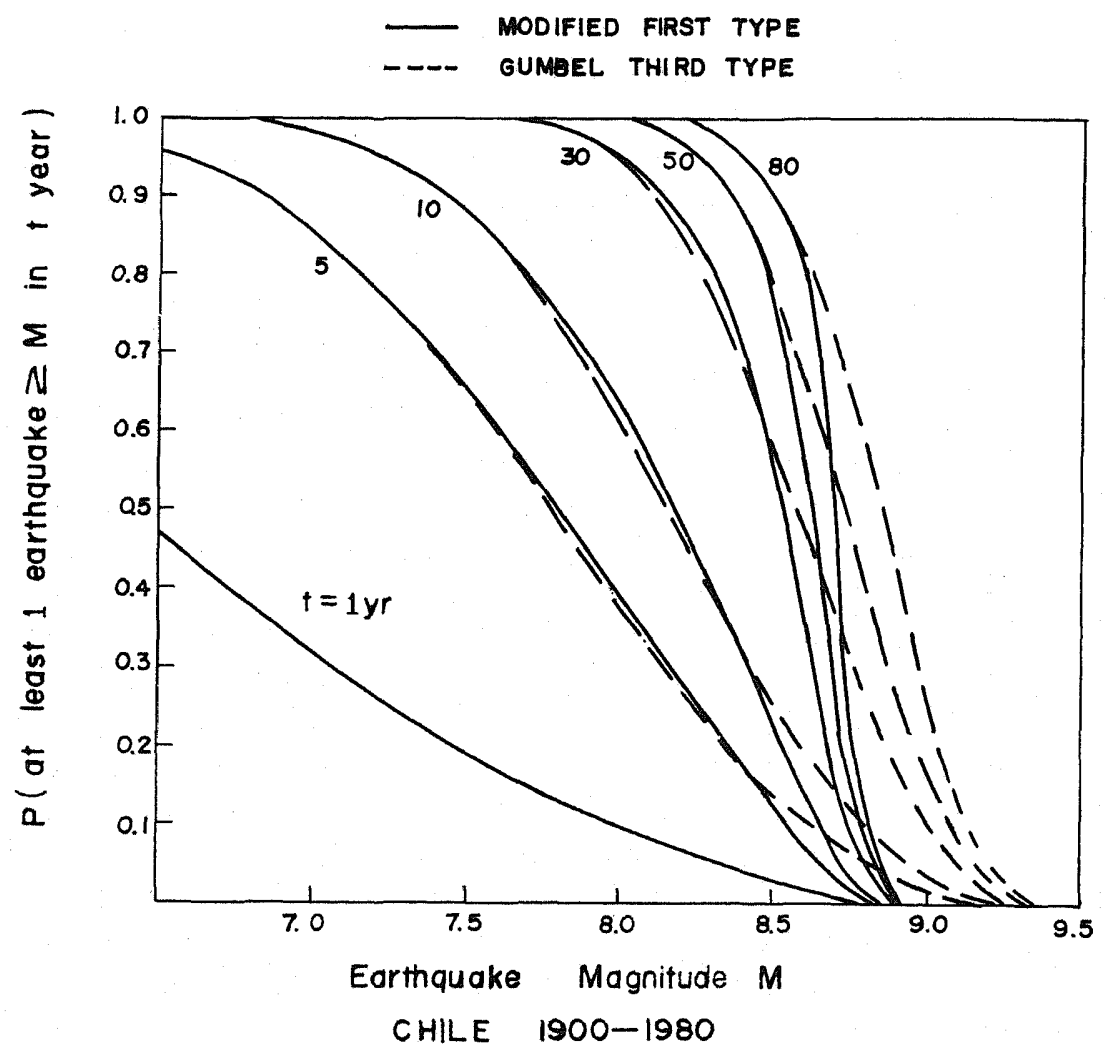

Fig. 3. Probabilities of exceeding magnitude $M$ in $t$ years in Chile. - and -- indicate the modified first type and Gumbel third type, respectively. 


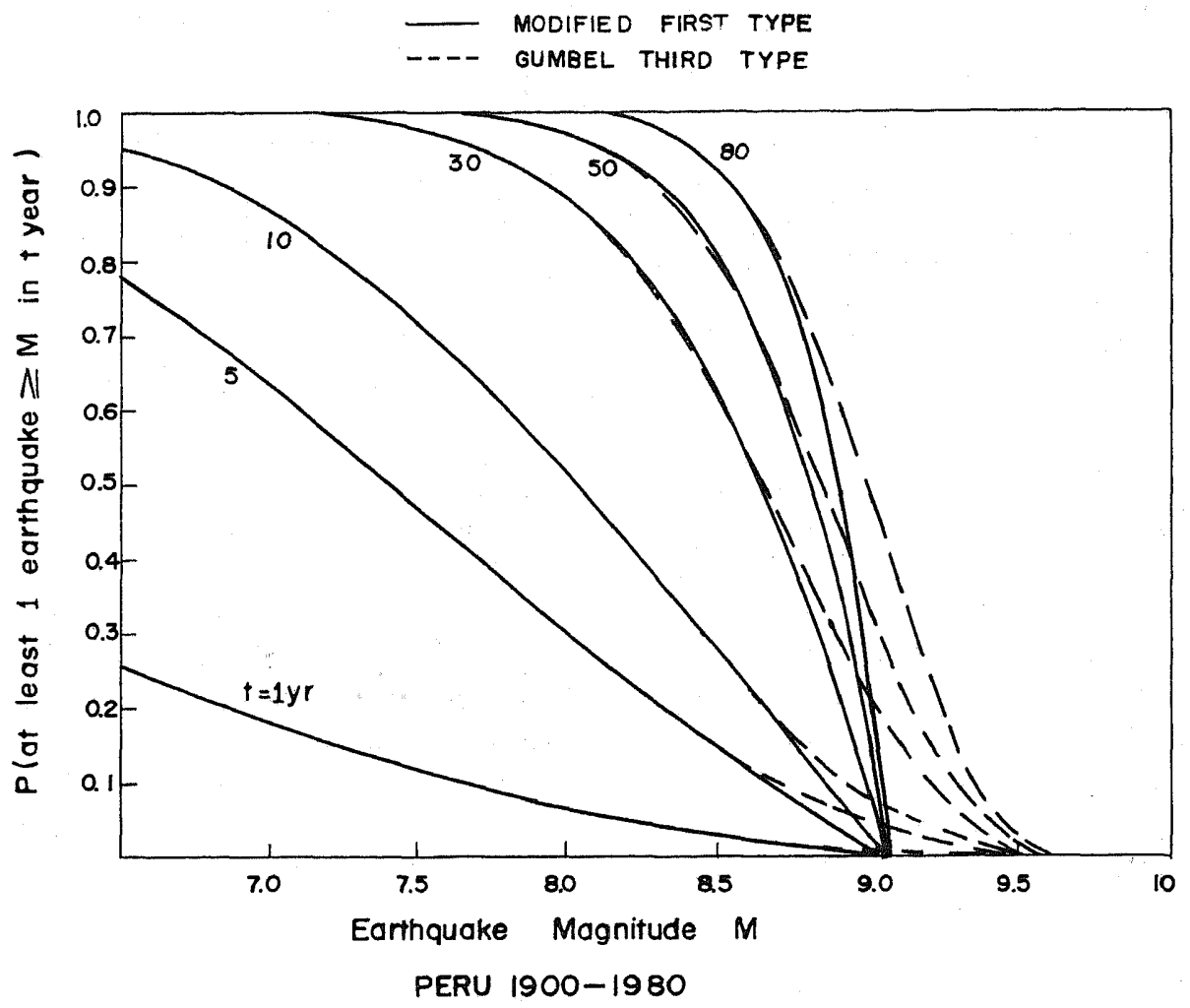

Fig. 4. Probabilities of exceeding magnitude $M$ in $t$ years in Peru. - and --- indicate the modified first type and Gumbel third type, respectively.

is obtained by subtracting from the statistical mean the number of earthquakes that occurred within the past $r$ years (see Table 3 ). In the Tokyo region, for example, 3.1 earthquakes of magnitude above 7.5 were predicted within $(r+20)$ years, while earthquakes of magnitudes 7.0, 7.1, and 7.9 actually occurred for the period 1920-1940. In the light of the assumption of constant accumulation and release of energy, it is natural that predicted earthquakes are approximately equal to actual ones. In the Guatemalan region, 2.3 earthquakes of magnitude above 7.0 were predicted within $(r+9)$ years. Earthquakes of magnitudes 6.4 and 6.5 actually occurred during the period 1970-1979. Similarly, the numbers of earthquakes that actually occurred and thuse predicted are found to be approximately equal in both the Managuan and Los Angeles regions.

The maximum magnitudes with a $10 \%$ exceeding probability within 20 years are found to be $8.3,8.8,8.1$, and 7.0 by the modified first type, and 8.5, 9.1, 8.2, and 7.0 by the third one for Tokyo in the period 1906-1979, the Guatemalan, Managuan, and Los Angeles regions, respectively.

Figures 3 and 4 show cumulative probabilities of Chile and Peru, where ex- 
treme values are equal to or exceed 6.5 in the next $t$ years (KIM, 1982). Assuming that earthquakes occur as the random variables of the Poisson model, one can easily notice that the cumulative probabilities increase as longer time intervals are selected. Probabilities of at least one earthquake $\geq 8.5$ in the next 80 years are one in both Chile and Peru, whereas the exceeding magnitudes that would occur at least once in the next 30 years are 7.5 and 8.0 for Peru and Chile, respectively. It is also found that the cumulative probabilities by the modified first type fall more steeply to the lower upper limit as compared with the third one.

\section{Discussion and Conclusions}

Figures 5 and 6 are shown only to demonstrate that the three methods, Howell (1981), modified first type, and Gumbel third type, lead to virtually identical results for world data. Howell (1981) suggested that the tanhfit equation fits better than the Gumbel third type equation, so it appears that saturation of the tanhfit equation occurs more suddenly than Gumbel's theory allows. However, the tanhfit equation can be applied to only large earthquakes in Howell. The modified first type, however, approaches saturation more suddenly than the third type through all sizes of earthquake data. Therefore, the modified first type gives results that are more reasonable than either the Gumbel third type or the tanhfit equation in the light of total part experience.

The extreme values of earthquakes are taken from NOAA data, which consist of $m_{\mathrm{b}}, M_{\mathrm{s}}$, and $M$. Geller and KANAMORI (1977) and KANAMORI (1977) have studied various magnitudes and energy releases of shallow-focus earthquakes and

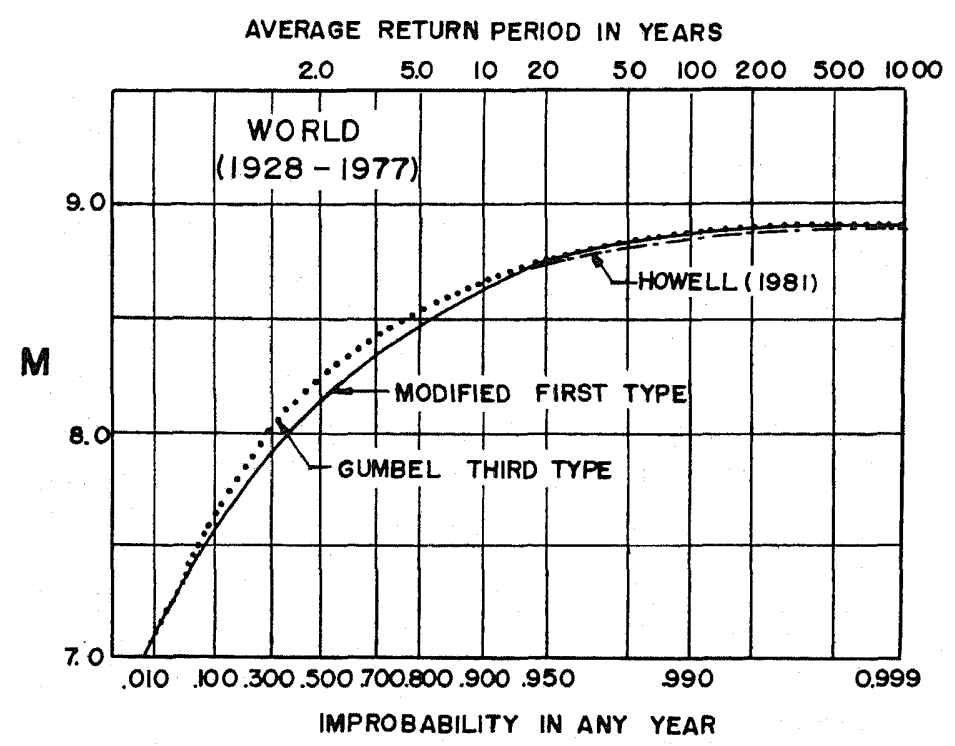

Fig. 5. Improbability of world $M$. 


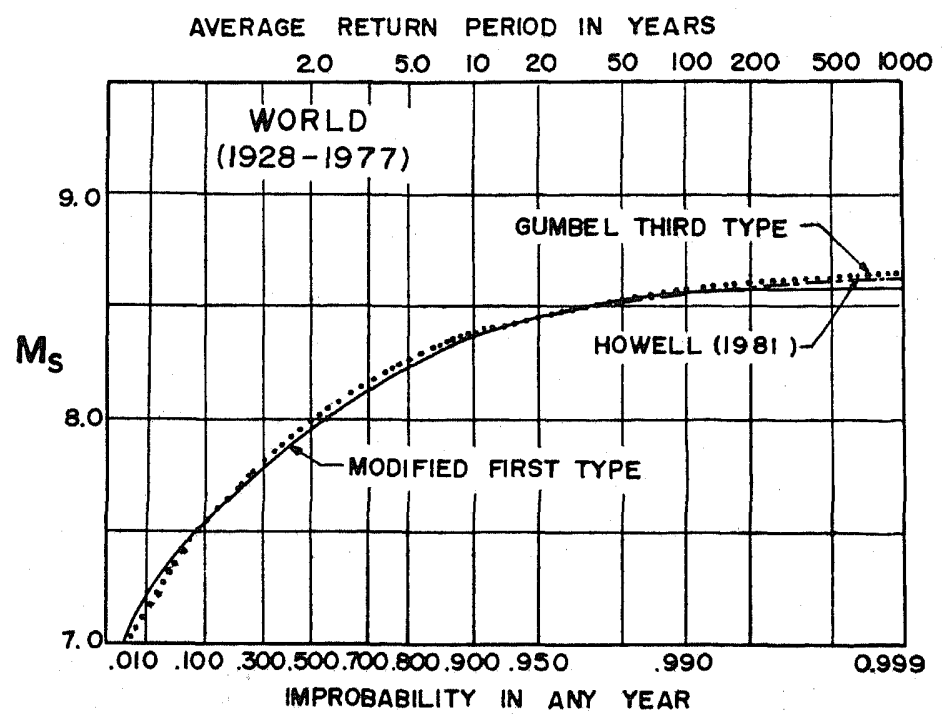

Fig. 6. Improbability of world $M_{\mathrm{s}}$.

found that there are some differences between $M_{\mathrm{s}}$ and $M$. In this study, we attempt to use the largest magnitudes values, usually $M$, among three magnitudes because all three magnitude types are not the same and not always reported for every earthquake. The probable error of individual maximum potential earthquakes has been assigned to within a half magnitude unit.

The modified first asymptotic function is formulated under the basis assumptions that (1) the prevailing nature of earthquake occurrence must be the same in the future, (2) a physical upper bound of the largest earthquake must exist, and (3) the magnitude distribution is independent of space and time. Seismologists, however, have found that the earthquake phenomena is nonrandom. Seismological observation suggests a dependence on at least two initial conditions, the size of the last great earthquake and the time elapsed since its occurrence. Seismic activities are also dependent on global tectonics or local geological structure such as faults. Therefore, the dynamic model and physical factors of earthquake occurrence should be considered important stochastic parameters in predicting the recurrence of great earthquakes.

\section{REFERENCES}

CH'EN, P'ei-shan and Pang-hui LIN, An application of theory of extreme values to moderate and long-interval earthquake prediction, Acta Geophys., 16, 6-24, 1973.

DudA, S. J., Secular seismic energy release in the circum-Pacific belt, Tectonophysics, 2, 409452, 1965.

Gumbel, E. J., Statistics of Extremes, Columbia University Press, New York, 375 pp., 1958.

Geller, R. J. and H. KANAMORI, Magnitudes of great shallow earthquakes from 1904-1952, 
Bull. Seismol. Soc. Am., 67, 587-598, 1977.

HATTORI, S., Seismic risk maps in the world (maximum acceleration and maximum particle velocity) (11)-Balkan, Middle East, Southeast Asia, Central America, South America and others, IISEE, 17, 33-96, 1979.

Howell, B. F., JR., On the saturation of earthquake studies, Bull. Seismol. Soc. Am., 71, 1401$1422,1981$.

KANAMORI, H., The energy release in great earthquakes, J. Geophys. Res., 82, 2981-2987, 1977.

KÁRNiK, V. and Z. HÜBERNOVÁ, The probability of occurrence of largest earthquakes in the European area, Pure Appl. Geophys., 70, 61-73, 1968.

KIM, So Gu, Linear inversion technique in seismic-acoustic processing, Korea Inst. Miner. Min. Eng., 15, 186-196, 1978.

KIM, So Gu, An application of the extreme value theory to the statistical prediction of earthquakes, Korea Geoscience Symposium, 86-99, 1981.

KIM, So Gu, Macrozonation and seismic risk maps, Proceeding of the 7th European Conference on Earthquake Engineering, September 20-25, 1982, Athens-Greece, Vol. 2, 43-50, 1982.

LomnITZ, C., Statistical prediction of earthquakes, Rev. Geophys., 4, 377-393, 1966.

SHAKAL, A. F. and D. E. WILLIS, Estimated earthquake probabilities in the North CircumPacific area, Bull. Seismol. Soc. Am., 62, 1397-1410, 1972.

UsAmI, T., Earthquakes and Information, Iwanami, Tokyo, 206 pp., 1974 (in Japanese).

WORLd MAP OF NATURal Hazards, Müchener Rückversicherungs-Gesellschaft, München, Federal Republic of Germany, 49 pp., 1978.

Yegulalp, T. M. and J. T. Kuo, Statistical prediction of the occurrence of maximum magnitude earthquakes, Bull. Seismol. Soc. Am., 64, 393-414, 1974. 